\title{
II. JAHRESBERICHTE.
}

\section{Xenophon.}

Zweiter artiket.

(Scliluss aus Pliilol. XXII, p. 681).

Die kleineren schriften Xenophons haben, obgleich hier die der erledigung harrenden fragen ziemlich zahlreich sind, verlıältnissmässig nur wenige bearbeiter gefunden. Wir nennen von den hierher gehörenden untersuchungen zuerst :

H. Beckhaus, De Xenophonteo qui fertur Agesilao. Diss. inaug. Berolini 1863.

Zwischen den entgegengesetzten ansichten derer, welche den Agesilaos für xenophonteisch und derer, welche ihn für ein untergeschobenes machwerk halten, nimmt diese abhandlung gewissermassen eine vermittelnde stellung ein, indem sie die schrift einem gleichnamigen enkel Xenophons, einem sohne des bei Nantinea gefallenen Gryllos, beilegt. Durch ziemlich umfangreiche untersuchungen bemüht sich der verfasser nachzuweisen, dass die sachlichen gründe, welche $C a$ u er (de fontibus historiae Agesilai) und $R$ a $n$ k $e$ (de vila Xenophontis: s. Plilol. XVIII, p. 245) gegen die ächtheit geltend gemacht haben, fast alle nicht zutreffend sind; dass die darstellungsweise im ganzen mit der xenophonteischen ühereinstimmt, dass das verhältniss, in welchem Xenophon zum könige Agesilaos stand, die alfassung einer solchen lobschırift wohl erklärlich macht, und dass endlich auch die wiederholung ganzer ahschnitte aus den Hellenicis nicht gegen die ächtheit sprechen. Was nun namentlich den ersten und den letzten punkt betrifft, so wird es allerdings schwer halten, gründe für oder gegen eine bestimmte ansicht zu finden, welche mehr als subjoctive, geschweige denn allgemein anzuerkennende geltung hätten. Wichtiger erscheint ein anderer punkt, auf den der verfitsser demnächst sein augenmerk gerichtet hat, näm- 
lich die sprache. Hier findet der verfasser wesentliche aluweicliungen von Xenophons sprachgebrauch, besonders in der mehr oder weniger läufigen anwendung der partikel $\mu \eta \dot{\eta} v$. Er giebt eine ülersiclit des vorkommens der partikelverbindungen $a \lambda \lambda \dot{\alpha}$

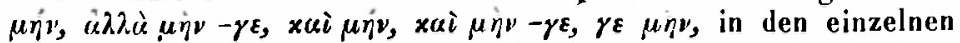
scliriften Xenophons, namentlich der letztern partikelverbindung vollständig, woraus sich ergielt, dass dieselbe in der Kyropädie mit ausnalıne des epilogs nur aclitmal, in der Anabasis viermal, in den Commentarien fünfmal, im Gastmalıl fünfmal, in den beiden ersten biichern der Hellenika, im Oekonomikos, im staat der Atliener, im Kynegetikos gar nicht gebrauclıt ist, wälırend sie sich im letzten capitel der Kyropädie viermal, in den fünf letzten büchern der Hellenika achtundzwanzigmal, im Agesilaos vierunddreissigmal, im staat der Iakedämonier dreiundzwanzigmal, in buch von den einkünften siebenzehnmal, im Hipparchikos zweiundzwanziginal, im buch von der reitkunst dreiunddreissigmal vorfindet. Der verfasser zielit hieraus den sclluss, dass in den letztgenannten sclıriften eine fremde hand zu erkennen sei. Die zusimmenstellung ist nun freilich liöchst ungenau; ich laabe, olıne gewälır der vollständigkeit, in der Kyropädie, alıgesehen von zwei falsclien angalien des verfassers (III, 10, 22? und IV, 5, 27) die partikelverbindung $\gamma \varepsilon \mu \eta_{y}$ an elf stellen angemerkt, in den beiden ersten büchern der Hellenika an zwei stellen (II, 3, 33 und 42), im Agesilaos an sechsunddreissig, im Gastmalıl an zelın, im stait der Lakedämonier an seclisundzwanzig, im buch von der reitkunst an seclisunddreissig, im Hipparchikos an dreiundzwanzig, in den fünf letzten lü̈clern der Hellenika an einunddreissig stellen; allein immerhin ist dieses resultat für den ersten anblick frappant. Wenn man dagegen bei nälıerer untersuclung findet, dass auch in den schriften, welche im ganzen jene art des iiberganges nur seltener liaben, jene partikeln sich doch an einzelnen stellen melırfach lıäufen, wie Kyrop. II, 1, 23 und 29; Hellen. 11, 3, 33 und 42; Conviv. VIII, 2; 3; 7; $13 ; 37$, ausserdem, dass die sclıriften, in denen sie lạ̈ufiger vorkommen, vielfach ein aneinanderreihen einzelner kurz dargestellter thatsachen aufweisen, so wird man den oben bezeichneten scliluss nicht unbedingt für sicher halten können, zumal da sich niclıt läugnen lässt, dass Xenoplion stark liervorliebende partikeln, wie ausser den angegebenen auch $\mu$ źt $\tau_{0 \iota}$ besonders liebt. lmmerhin ist die sache wolı einer genaueren untersuchung werth.

Der verfasser meint nun weiter, jener enkel Xenophons, von dem wir schon oben sprachen, lialse seines grossvaters werke entweder herausgegeben oder vorhandene in materiale einleitungen, epiloge und viele sentenzen nach rhetorischer weise hinzugefügt. Diesen enkel aljer, den auch Grote in einer stelle des Diogenes Laertios zu erkennen glaubte, laat der verfasser in Plıotii biblioth. 
cod. 260 zu finden gemeint, wo es vou Isokrates heisst: $I_{\varepsilon-}$

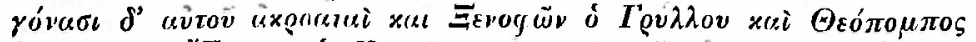

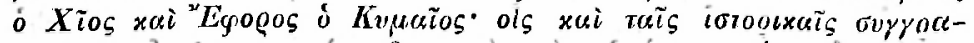
rpais rogù

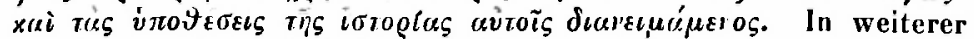
ausfulırung dieser hypothese sucht der verfasser auch an einzelnen stellen der betreffenden scliriften eigenthümlichkeiten des Isokrates nachzuweisen. Ich halte diese hypothese für nichts weniger als glücklich. Zunächst bleiltt unentschieden, ob der jüngere Xenophon seine bearbeitungen als seine oder seines grossvaters arbeiten herausgegeben hat. Wäre das erstere der fall, so fragt man, wie das ganze alterthum in dem irrtlum befangen sein konnte, jene sclıriften für arbeiten des älteren Xenophon zu halten, so dass erst Demetrios Magnes die vermuthung aussprach, dass ein paiar von den kleineren schriften nicht von demselben verfasst seien. Hätte er sie als werke seines grossvaters herausgegeben, so drängt sich die frage auf, wie Plıotius in so später zeit und zwar ganz allein die notiz erhalten habe, sie seien bearbeitungen eines jüngeren Xenophon. Endlich angenommen, es sei dies möglich, so bleibt es unbegreiflich wie er den jüngeren Xenophon als historiker mit Theopomp und Ephoros auf eine linie stellen konnte, während die vom verfasser ihm zugeschriebene arbeit doch nimmermelır als arbeit eines historikers angesehen werden darf, algesehen davon, dass dies mit der etwas sonderbaren notiz über die aufgaben, die lsokrates seinen schülern gestellt haben soll, nicht in einklang zu bringen ist. Ich bin aber fest überzeugt, dass Photius wirklich den älteren Xenophon gemeint hat; denu mit dem Ephoros und Theopomp würde er einen angeblichen historiker, von dem im alterthum kein mensch eine silbe erwähnt, sicher nicht zusammengestelit haben, zunal da von den liundert schülern des lsokrates (Vita $X$ Orr. p. $837 \mathrm{C}$ ) manclier andre historiker niedern ranges mit viel grösserem rechte häitte erwähnt werden können. Welche quelle dem Plotius den Isokrates als lelirer Xenophons genannt hat, und ob nicht dahei eine nicht ungewöhnliche verwechselung des Isokrates mit dem Sokrates zu grunde liegt, dürfte nicht leicht nachweisbar sein.

Ueber das buch vom state der Lakedämonier hat CoLet Novae Lectt. p. $705 \quad-724$ ausführlich gehandelt. Er hält es allerdings für vielfich verstümmelt und verdorben, aber doch für acht, sowohl weil in der schreibart sich nichts findet, was dem Xenophon fremd wäre, als auch weil gerade die irrthümer in den dingen, welche entfernter liegende zeiten betreffen, auf eine al,fassungszeit hindeuten, in welcher die historische kritik noch nicht die wahrheit der tradition zum gegenstande ihrer untersuchungen gemacht liatte. Das zeugniss des Demetrios Magnes, der zuerst das buch für nicht xenophonteisch erklärte, achtet 
Cobet nicht und weist nach, dass auch das alterflum nichts darauf gegelien. Er ist nun der ansicht, dass Xenoplion in dem buche nicht was er selbst geselien, sondern die alten einrichtungen, wie er sie aus den erzälılungen des Agesilios und anderer kannte, dargestellt und nur einzelnes aus eigner erfilirung entnommene beigemischt habe, woraus denn einzelne widersprïche in seinen angaben hervorgegangen seien. Die alfassung selbst gehört in eine zeit, in welcher die macht der Lakedämonier noch nicht ganz gebrochen war, wie dies einige stellen des bu. ches zu erkennen geben (vgl. 14, 4 und 5). Zum schlusse zeigt Cobet an einer reihe dem Xenopbon eigenthümlicher ausdrücke die übereinstimmung des buches mit Xenophon's schreibweise, so wie an einzelnen sentenzen die übereinstimmung mit seinem charakter.

Ein gleiches urtheil faillt Cobet p. 706 über die ächtheit der schrift vom stante der Athener, die freilich durchweg so verstümmelt und verdorben sei, dass man keine aussicht habe, den wahren zweck derselben zu ergründen. Dennoch zieht er aus einigen einzellieiten den schluss, dass das vorhandene wohl aus einem dialoge herriihren könnte, in welchem sich zwei personen in der art über den athenischen statut unterhielten, dass der eine denselben lieftig tadelte, während der andere die riclitigkeit dieses tadels anerkannte, aber zeigte, dass die demokratie nicht anders als unter den vorhandenen formen bestehen könnte. Die spuren des dialogs findet Cobet nimentlich in den stellen, welche eine directe anrede an eine zweite person enthalten. - Eine wesentlich verschiedene ansicht findet sich ausgesprochen in :

C. Morel, Ruaestiones de libello qui dicitur Xenophontis de republica Atheniensium. Dissert. inaug. Bonnae 1858.

Der erste abschnitt dieser schrift beschäftigt sich damit, nachzuweisen, dass die in rede stehende angeblich xenophonteische schrift etwa um das frülijahr 424 v. Chr. verfasst worden sei. Der verfasser gründet diese zeitbestimmung zunäclıst auf die von Roscher aufgestellte insicht, die schrift sei ein brief eines athenischen optimaten an einen Lakedämonier, worin derselbe jenem die gründe mittlıilen wollte, weshalb die verfassung Athens nicht leicht zu stürzen sei. Eine solche mittheilung passe aber nur für die zeit, in welcher nach Thuk. 4, 55 die La. kedämonier an meisten an einem glücklichen erfolge des krieges verzweifelten. Allein die richtigkeit dieser ansicht von dem zwecke der schrift dürfte schwer zu beweisen sein, da vieles in dem buche enthalten ist, was äber jenen zweck weit hinausgeht, da doch offenbar das ganze darauf abzielt, dass die einzelnen statlichen einrichtungen der Athener mit der einmal gewällten verfussung, möchte diese nun im ganzen sein, wie sie wollte, im einklang stehen: damit ist freilich nicht gesagt, dass die ansicht des verfassers von der zeit der alffissung entschieden unrichtig ist; es 
mochte vielleicht ziemlich wahrscheinlich sein, dass die sclırift ungefalır aus jener zeit herrülırt, nur ist eine so genaue bestimmung, zumal auf grund jener ansicht, unmöglich, und auch die ejinzelnen stellen, die der verfasser noch anfülırt, ohne beweisende kraft.

Lässt man diese zeithestimmung zu, so kann Xenophon nicht der verfasser des bucbes sein, eine annahme, die der verfasser der abluandlung durch eigenthiimlichkeiten in der darstellung und im sprachgebrauche meist nur durch verweisung auf frühere behandlungen des gregenstandes zu begründen sucht, die wir deshalb auf sich berulien lassen können. Wer der schreiber dieses angeblichen briefes gewesen, lässt der verfasser unentschieden, sucht aber durch vergleichung mit anderen stellen, namentlich aus dem buche über die einkünfte zu zeigen, dass Xe. nophon die schrift gekannt, und meint, dieselbe sei walırscheinlich nach Xenophons tode unter seinen papieren gefunden und so für sein werk gehalten worden, eine lyypothese, die jedes anlialtes entbelirt.

Gefördert wird für das verständniss des bucbes durch alle diese vermuthungen nichts. Was der verfasser zum schluss auf wenigen seiten ïber die lïcken, umstellungen und interpolationen des textes beibringt, ist unbedeutend.

Specimen literarium inaugurale exlibens Xenophontis librum de vectigalibus cum commentario, quod - eruditorum examini submittit J. C. van Deventer. Trajecti ad Rhenum 1851 .

Auf p. 1--28 ist der text abgedruckt, unter welchem sich einzelne bemerkungen über aufgenommene conjecturen oder vorgeschlagene änderungen befinden. Demnächst folgt p. 29-64 eine abhandlung über den plau und die abfassungszeit der sclırift. Der verfasser vertheidigt seine ansicht, dass das buch in der nächssten zeit nach der schlacht bei Mantinea, etwa 361 v. Chr. geschrieben sein müsse besonders mit rücksicht auf Böckh, der (Staatshaush. 1 p. 778 ff. der zweiten ausg.) annahm, dass die schrift unmittelbar nach beendigung des bundesgenossenkrieges 0l. 106, 1 verfusst sei. Zunächst meint er, die worte 5, 12

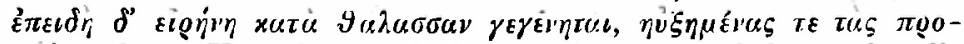
oodovs lätte Xenophon nach dem bundesgenossenkriege, der die Athener aller bundesgenossen und einkünfte beraubte, nicht schreiben können. Allein man sieht leicht, dass diese behauptung falsch ist, denn sicber blülıte mit dem albschlusse des friedens auch der handel wieder empor und erhölıte durch die eingehenden zolle die einkünfte des statates, wenn auch keine beiträge von bundesgenossen einliefen, und dass Xenophon dergleichen einkünfte im sinne hat, die überhaupt im frieden eingehen, im kriege ausbleiben, lehirt der zusammenlang. Dazu kommt, dass man die

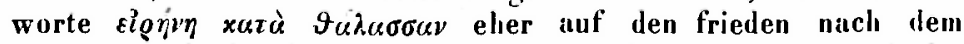
seekriege mit den bundesgenossen, als auf den frieden nach der 
schlacht hei Mantinea, der hauptsächlich einen landkrieg beendete, deuten kann. Die zweite bemerkung, dass Xenophon in dieser zeit den makedonier Plhilipp nicht unerwälınt gelassen, und die Athener nicht zum frieden würde aufgefordert haben, während sie mit allen kräften den gemeinsamen feind bekämpfen mussten, ist werthlos gegenüber dem ganzen inhalte des buches, der nicht gerade tiefen politischen scharfblick bewälırt (vgl. Böckh a. a. 0 . p. 781 ff.). Drittens bemerkt der verfasser, Xenophon spräche melırmals so, als ob es noch bei den Athenern stünde, die bundesgenossen durch milde an sich zu fesseln oder durch ungerechten druck sich zu entfremden; dies passe auf die angenommene zeit nicht. Welche stellen der verfasser im sinne hatte, hat er nicht angegel,en, lässt sich auch nicht errathen, wälırend 5, 5 ì r $\dot{\eta}_{\gamma \varepsilon-}$

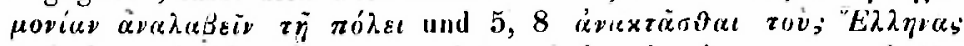
auf eine zeit linweisen, wo Atlien keine bundesgenossen hatte. Der versuch diese ilım entgegenstehende sclıwierigkeit dadurch zu lösen, dass Xenophon hier nicht die liegemonie im allgemeinen, sondern nur die in ilırem frülıeren umfange gemeint habe, kann nicht als gelungen augesehen werden. Die argumente endlich, die der verfusser für seine ansicht geltend macht, sind eluenfalls schwach. Die bezielıung von 3, 7 auf den zug nach Arkadien und auf die schlacht bei Nantinea konnte der sclıriftsteller eben so gut noch 356 wie 361 v. Chr. nelimen, da gewiss auch im ersteren jahre die damals gemachten anstrengungen den Athenern

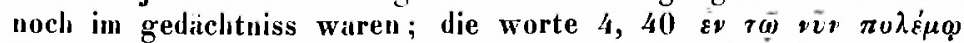
können ebensogut auf den bundesgenossenkrieg wie auf den durch die schlacht bei Mantinea abgeschlossenen krieg bezogen werden, und wenn auch die 5, 8 erwälınte $\tau \alpha \rho \alpha \chi \dot{\eta}$ in Griechenland nach Hellen. VII, 5, 27 unmittelbar nach der scblacht bei Mantinea eintrat, so bestand dieselbe gewiss noch 356, ja noch länger.

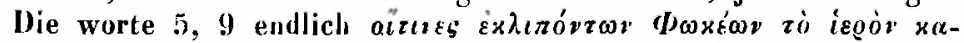

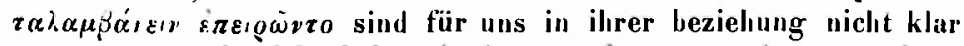
genug, um mit sicherheit mit dem verfusser annelımen zu kön. nen, dass die zeit gemeint sci, wo die Thebaner nach unterwerfung der böotischen städe auch die Phokcer angriffen. Eine ganz andere deutung dieser stelle hat Colset Novae Lectt. p 755-767 versucht, um dadurch die ansicbt Böcklis, welcher er sich vollständig anschliesst, noch mehr zu stiitzen. Er bezieht unbedingt izgòv auch zu érhasózzov, das er nach der iublichen bedeutung des wortes vom gezwungenen aufgeben einer besetz. ten position erklärt. Das factum, das alsdann damit gemeint wäre, sucht er durcl folgende betraclitung zu finden. Die vollständige besiegung der Phokeer fällt sicher 0 !. 108, 3 gegen ende des jallres, der anfiang des leiligen krieges, der nach allgemeiner angalse zelın jahre gewährt hat, also 01.1062 ; demnach batten die Plokeer den delphischen tempel ein jahr nach alfiassung unsers bucles in besitz genommen, und die in unserer 
stelle angedeutete aufgabe des besitzes kaun sich darauf nicht beziehen. Nun heisst es aljer bei Diodor. 16, 14, der tempel sei 0l. 105, 4 von den Phokeern ausgeraubt worden und der nun folgende krieg habe elf jahre gedauert, während 16, 23 die dauer desselloen auf neun jahire angegeben wird; auch Pausan. 10, 2, 3 nennt 0l. 105, 4 als das jahr, wo die Phokeer den tempel in besitz genommen. Colet nimmt nun an, diese angabe sei richtig, die Plokeer hätten jedoch aus irgend cinem grunde bald darauf den besitz anfgegeben und nun die Thebaner den in unsrer stelle erwähnten versuch gemacht; inzwischen sei über die Plokeer die bekannte unerschwingliche geldstrafe verläingt worden, und sie hätten nun zum zweiten male den rempel in besitz genommen und jetzt ausgepliindert. In folge dessen halse man allgemein angenommen, dass der krieg erst mit dieser zeit begonnen, wälirend genauere historiker, aus denen Diodor und Pausanias schöpten, jene erste besetzung als veranlassung des krieges aufführten. Ich glaube, dass diese scharfsinnige hypothese der beachtung wohl werth ist.

Die zweite hälfte des buches von Deventer (p. 6j̃-118) bildet cin commentar kritischen und exegetischen iubalts, von dem namentlich der sachliche theil nicht ülıer das allgemein bekanrte hinausgeht.

Blicken wir zum schluss auf die leistungen zurïck, die wir licer allmählig vorgeführt haben, so müssen wir zwar anerkennen, dass in dem letzten jahrzehnt für Xenophon namentlich dadurch viel geschehen ist, dass wir von I)indorf ausgaben von allen grösseren werken erhalten haben, die den beutigen anforderungen in ihrer art genïgen, dass aber auch noch viele fragen der losung, viele schwierigkeiten und unsicherheiten im ganzen wie in einzelheiten eingreliender hehaudlung warten.

Berlin.

B. Bùchseuschütz.

\section{Zu Plutarchos.}

Plutarch. Cat. mai. 8 erwähnt den ausspruch Cato’s oi óna

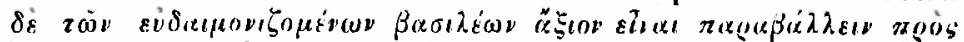

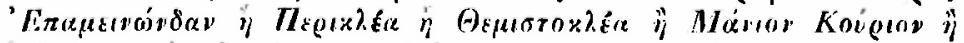

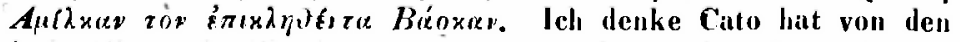
drei vïkern je einen mann genannt, der nach scinem herzen war, Epaminondas, Curius Dentatus, IIamilkar Barkas, und tilge

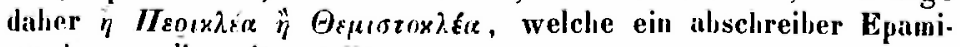
nondas an die seite stellte.

Greifswald.

A. Schaefer. 